\title{
Determination of Pesticide Residues on the Surface of Fruits Using Micro-Raman Spectroscopy
}

\author{
Yande $\mathrm{Liu}^{1,2}$ and Tao $\mathrm{Liu}^{1}$ \\ ${ }^{1}$ School of Mechanical and Electronical Engineering, \\ East China Jiaotong University, Nanchang, China \\ ${ }^{2}$ Institute of Optics-Mechanics-Electronics Technology and Application (OMETA), \\ East China Jiaotong University, \\ Nanchang, China
}

\begin{abstract}
A simple, rapid and environmentally friendly method was developed for micro-Raman spectroscopy determination of pesticide residues on the surface of fruits. Raman spectra of fruits, pesticides and pericarps sprayed by pesticide solutions were acquired using a laser power of $14 \mathrm{~mW}$ at excitation wavelength of $780 \mathrm{~nm}$. From the Raman spectra, the residual pesticides could be distinguished and determined through the characteristic Raman peaks. The overall results indicted that micro-Raman spectroscopy is a potential tool to determine the pesticide residues on the surface of fruits for fruit quality and safety control.
\end{abstract}

Keywords: micro-Raman spectroscopy, fruit, pesticide residue.

\section{Introduction}

Pesticides are defined by the United Nations Food and Agricultural Organization (FAO) as substances or mixtures intended to prevent, destroy, repel or mitigate any pest, including insects, rodents and weeds [1]. Currently, pesticides play a critical role in protecting fruit crops. However, large amount of pesticides are used in fruits production making it unsafe and endangering humans and animals. One of the most common used pesticides are the organophosphates, which kill insects and mites by attacking the central nervous system, and consequently pose a threat to human beings and animal lives [2]. Nowadays, fruit safety and quality has been attracted more and more attention. In many countries, strict tolerance levels are set up to ensure public safety.

The current analysis methods for determining the residual pesticides in fresh fruits are based on chemical analysis, such as liquid chromatography-mass spectrometry (LC-MS) [3], liquid chromatography-tandem mass spectrometry (LC-MS/MS) [4, 5], gas chromatography-mass spectrometry (GC-MS) [6], capillary electrophoresis-mass spectrometry (CE-MS) [7] and ultra-high-performance liquid chromatography (UHPLC) [8], etc. However, all of the methods mentioned above are time-consuming, not environmentally friendly and inconvenient enough for the process of sample preparation. Hence, it was very necessary to develop a simple, rapid and low cost method for the determination of pesticide residuals in fruits. 
Raman spectroscopy is a fast technique compared to classical chromatography. It is a powerful analytical tool that provides wonderful advantages including non-destructive, low cost and ultrasensitive characterization down to single molecular level [9]. Shende et al. [10] used surface-enhanced Raman spectroscopy (SERS) to analyze pesticides on fruit surfaces. Zhou et al. [11] recorded several spectra of fruits with pesticides using near infrared Fourier transform Raman spectroscopy (FT-Raman). Several Raman spectra of some fruits and pesticides on the surface of fruits were acquired and compared with at two excitation wavelengths of 514.5 and $1064 \mathrm{~nm}$ by Zhang et al. [12].

Micro-Raman has its unique characters compared to other Raman techniques, since it allows analysis of materials with a spatial resolution of several microns focused by an optical microscope. It has been developed for a rapid, environmentally friendly and low cost generation procedure, and has been applied in many fields, such as gems [13, 14], fibers $[15,16]$ and geology $[17,18]$, etc. In this paper, a new attempt to determine the residual pesticides on the surface of fruits using micro-Raman spectroscopy was reported. The objective was to record the Raman spectra of fruits and pesticides in order to study the feasibility of using micro-Raman spectroscopy to determine the residual pesticides on the surface of fruits.

\section{Materials and Methods}

\subsection{Apparatus}

A Nicolet DXR Raman Microscope (Thermo Fisher Scientific Corp., Madison, WI, USA), equipped with a liquid nitrogen cooled CCD detector and a $14 \mathrm{~mW}$ maximum power diode laser, that emits at $780 \mathrm{~nm}$, was employed for confocal micro-Raman spectra acquisition using $2 \mathrm{ml}$ standard glass chromatographic vials (12 $\mathrm{mm} \times 32 \mathrm{~mm}$ ) as sample cells. The laser was focused within the sample using an inverted microscope setup equipped with a $10 \times$ ultra long working distance objective. The scattered signal was then recorded at a $180^{\circ}$ backscattering geometry and dispersed by a single monochromator using a 400 grooves/mm diffraction grating and a laser line filter used as a beam splitter to lead the laser beam into the microscope assembly and also to reject the Rayleigh scattered light on the returning path. Spectrometer was controlled with OMINIC software (Thermo Nicolet Corp., Madison, WI, USA).

\subsection{Sample Preparation and Chemicals}

The samples, including apples, pears and oranges fruits in the experiment, were purchased directly from the Nanchang fruit market. The chemicals, dimethoate standard $(O, O$-dimethyl- S-(N-methylcarbomoylmethyl) phosphorodithioate) solid $(99.1 \% \mathrm{w} / \mathrm{v})$, chlorpyrifos standard (O,O-diethyl-O-(3,5,6-trichloro-2-pyridyl) phosphorothioate) solid $(99.5 \% \mathrm{w} / \mathrm{v})$ and malathion standard ( $S$-(1,2-dicarbethoxyethyl)- $O, O$-dimethyl dithiophosphate) solution $(99.1 \% \mathrm{w} / \mathrm{v})$ were purchased from bzwz corporation (Beijing, China). Two emulsifiable concentrate commercial pesticide formulations, chlorpyrifos $(40 \% \mathrm{w} / \mathrm{w})$ and malathion $(40 \% \mathrm{w} / \mathrm{w})$ were obtained directly from the Nanchang pesticide market.

Before recording the micro-Raman spectra, all the fruits were cleaned carefully. Firstly, fruits were flushed several times with fresh water to get rid of the dirt left on the 
surface, and then wiped with cotton and alcohol. After that, small pieces of pericarps from the cleaned fruits were taken off, and then sprayed by a little amount of pesticide formulations. At last, filter papers were used to wipe off any left over pesticides from the pericarps, and let the samples dry naturally.

\subsection{Micro-Raman Procedure}

When it was time to record the micro-Raman spectra, the resolution and accumulating time were fixed at $1.93 \mathrm{~cm}^{-1}$ and 100 scans per spectrum. The micro-Raman spectra of clean pericarps of apples, pears, oranges and four standard pesticides were collected, firstly. These spectra were used as standards in the comparison database. Then the spectra of pericarps sprayed by the pesticide formulations were collected and compared with those in the database to identify the trace amount of pesticides on the surface of fruits. All the spectra mentioned above were carried out in chromatographic glass vials from 3390 to $110 \mathrm{~cm}^{-1}$ with the diode laser power employed fixed at $14 \mathrm{~mW}$.

\section{Results and Discussion}

\subsection{Raman Spectra of Fruits}

Based on the measuring conditions mentioned above, the micro-Raman spectra of clean pericarps of apple, pear and orange fruits are shown in Fig. 1. Each of fruits has its characteristic peaks. Oranges mainly have the Raman peaks at 1530, 1155 and 1005 $\mathrm{cm}^{-1}$ attributed to $\mathrm{C}=\mathrm{C}$ stretching, $\mathrm{C}-\mathrm{C}$ stretching and $\mathrm{C}-\mathrm{CH}_{3}$ bending of carotene [19]. However, both apple and pear fruits have combined peaks around 1600, 1439, 1136 and $850 \mathrm{~cm}^{-1}$. It is estimated that these peaks are due to the combination of Raman scattering peaks by nourishment compositions of apple and pear. Because each of them contains very complicated compositions as there are usually different amount of water, sugar, vitamin, fat, protein, amino acid, as well as other components and elements such as calcium, iron and zinc, etc. At present, these fruits still can not be identified from the Raman peaks, but it has differences in the peaks attributed to the different compositions.

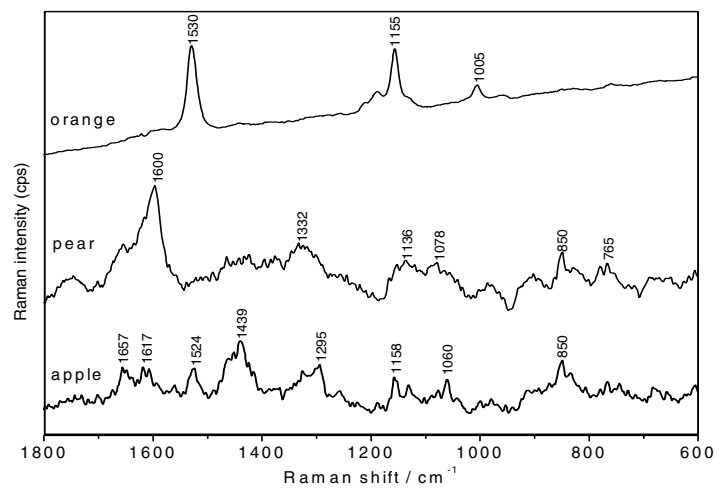

Fig. 1. Raman spectra of orange, pear and apple fruits 


\subsection{Raman Spectra of Pesticides}

Raman spectra of three standard pesticides, including dimethoate, chlorpyrifos and malathion, are shown from Fig. 2 to Fig. 4 in the 3390-110 $\mathrm{cm}^{-1}$ region. All pesticides have the same structure with $\mathrm{P}=\mathrm{S}$ bonds, which display a strong band in the $600-700 \mathrm{~cm}^{-1} \mathrm{re}-$ gion. This band is clearly distinguishable for compounds without benzene rings, but several of the vibrations due to the benzene ring overshadow it as in chlorpyrifos spectrum, where two overlapping bands appear at 630 and $676 \mathrm{~cm}^{-1}$ in Fig. 2 [20]. The most intense bands in the chlorpyrifos Raman spectrum are those present at 340 and $630 \mathrm{~cm}^{-1}$ due to $\mathrm{N}$-cyclopropyl bending and ring deformation, respectively. Other less intense bands located at $158,676,1276$ and $1569 \mathrm{~cm}^{-1}$ are due to P-O vibration, ring breathing, C-H bending and $\mathrm{C}=\mathrm{C}$ stretching. $\mathrm{C}-\mathrm{H}$ stretching modes are observed from 2800 to $3100 \mathrm{~cm}^{-1}$.

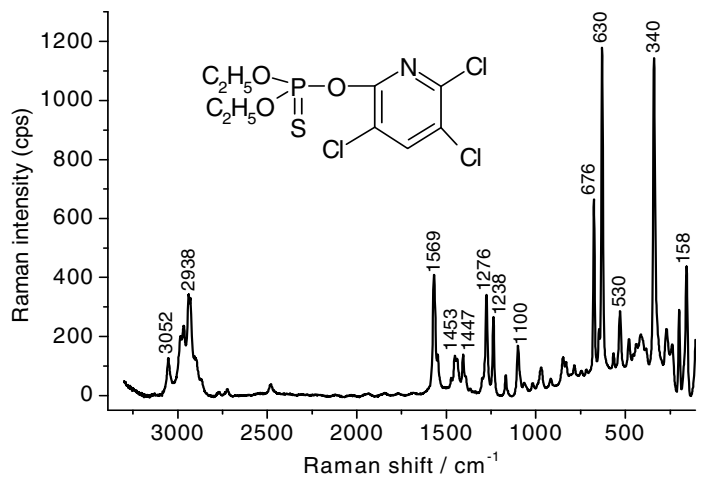

Fig. 2. Raman spectrum and molecular structure of chlorpyrifos

In Fig. 3 of dimethoate Raman spectrum, $\mathrm{P}=\mathrm{S}$ bond displays a strong band at $696 \mathrm{~cm}^{-1}$. The most intense band in the spectrum is located at $575 \mathrm{~cm}^{-1}$ due to C-S vibration. Other less intense bands located at 171, 494, 768, 903, 1156, 1330 and $1640 \mathrm{~cm}^{-1}$ are due to P-O vibration, P-S vibration, S-P-O backbone mode, C-C backbone stretching, $\mathrm{C}-\mathrm{C}$ stretching, $\mathrm{N}-\mathrm{H}$ in-plane bending and symmetric $\mathrm{C}=\mathrm{O}$ stretching. The intense bands appearing around $2936 \mathrm{~cm}^{-1}$ are due to $\mathrm{CH}_{2}$ and $\mathrm{CH}_{3}$ deformation.

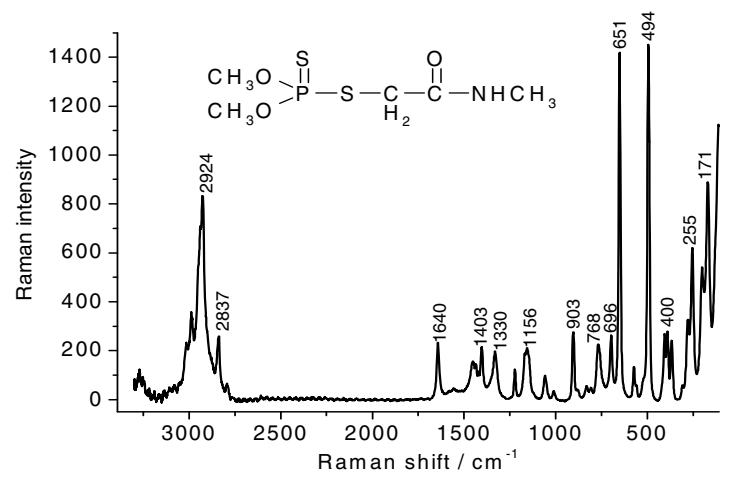

Fig. 3. Raman spectrum and molecular structure of dimethoate 
In Fig. 4 of malathion spectrum, $\mathrm{P}=\mathrm{S}$ bond displays a strong band at $652 \mathrm{~cm}^{-1}$. The bands located at 159, 496, 859, 1022, 1110 and $1733 \mathrm{~cm}^{-1}$ are due to P-O vibration, P-S vibration, $\mathrm{C}-\mathrm{O}-\mathrm{C}$ stretching, $\mathrm{C}-\mathrm{H}$ in-plane deformation, $\mathrm{C}-\mathrm{C}$ stretching and $\mathrm{C}=\mathrm{O}$ stretching [21]. The intense bands appearing in 1451 and 2800 to $3100 \mathrm{~cm}^{-1}$ are due to $\mathrm{CH}_{2}$ and $\mathrm{CH}_{3}$ deformation and $\mathrm{C}-\mathrm{H}$ stretching.

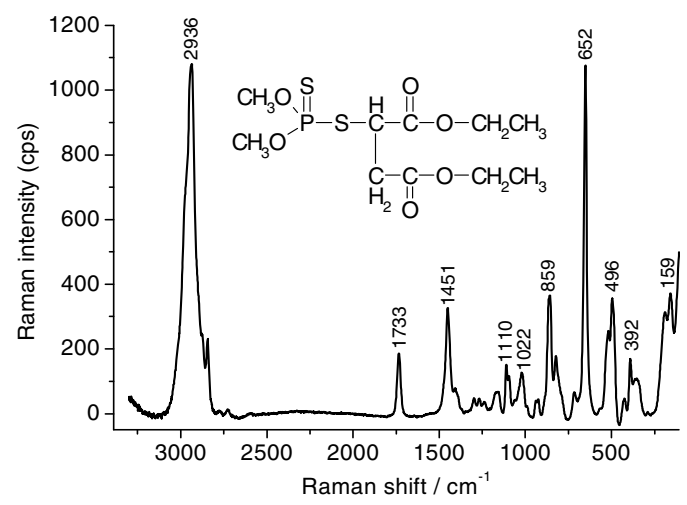

Fig. 4. Raman spectrum and molecular structure of malathion

From the three figures mentioned above, each of pesticides could be distinguished using their characteristic peaks shown in Table 1. These bands could be employed for determining pesticide residues on the surface of fruits.

Table 1. Characteristic peaks of chlorpyrifos, dimethoate and malathion

\begin{tabular}{ll}
\hline Pesticide & Characteristic peaks $\left(\mathrm{cm}^{-1}\right)$ \\
\hline Chlorpyrifos & $1569,1276,676,630,340$ \\
Dimethoate & $1640,651,494$ \\
Malathion & $1733,1451,652$ \\
\hline
\end{tabular}

\subsection{Raman Spectra of Pesticide Residues on the Surface of Fruits}

Acquirements of Raman spectra of fruits with pesticides were performed with the same measuring conditions as before. Fig. 5 and Fig. 6 show the Raman spectra of chlorpyrifos and malathion formulations residues on the surface of oranges. From Fig. 5, it can be obviously seen that the spectrum of chlorpyrifos residue on the surface of orange not only contains the strong peaks of orange itself, but also contains the characteristic modes of chlorpyrifos which located at 676,630 and $340 \mathrm{~cm}^{-1}$. Similarly, in Fig. 6, the characteristic modes of malathion at $1451,859,652$ and $496 \mathrm{~cm}^{-1}$ can be seen in the spectrum of its residue on the surface of orange. Based on the results, it was concluded that the method of using micro-Raman spectroscopy could be utilized for automatic and intelligent determination of the pesticide residues on the surface of fruits. 


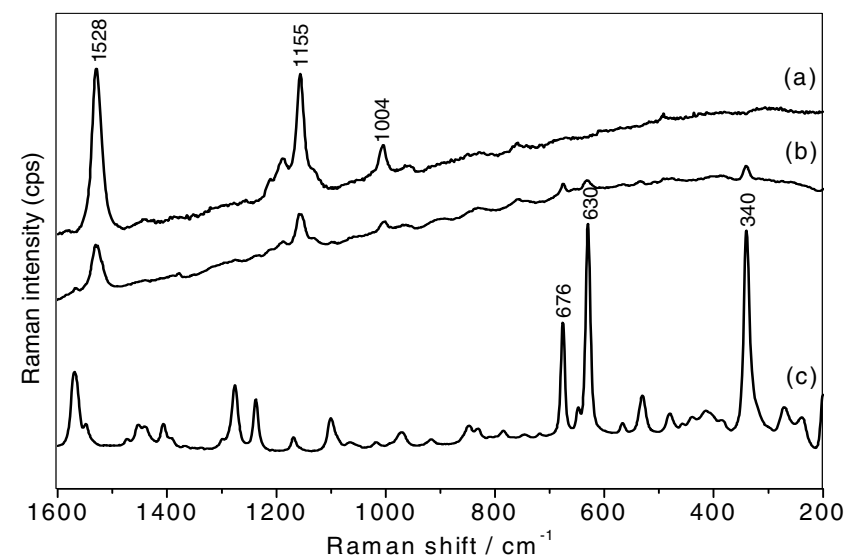

Fig. 5. Raman spectra of (a) orange's pericarp, (b) chlorpyrifos formulation (40\% w/w) left on the surface of orange and (c) standard chlorpyrifos solid

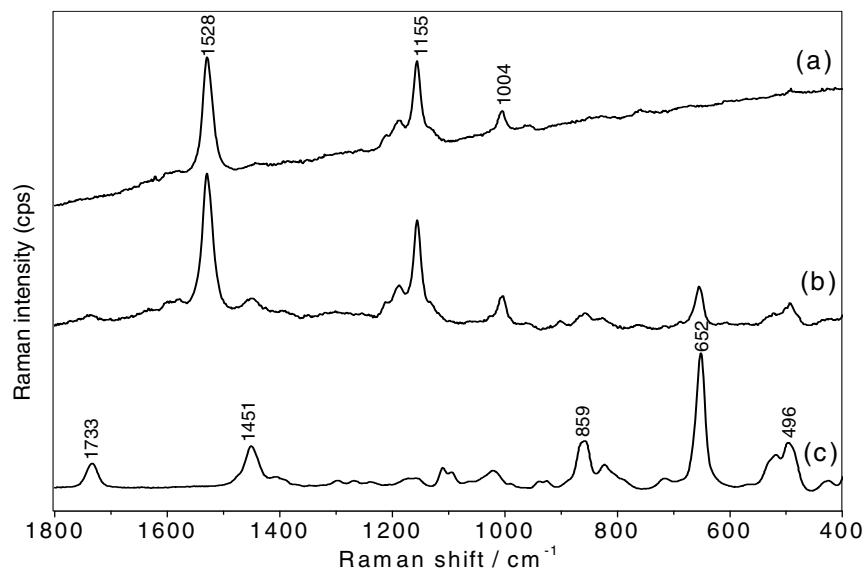

Fig. 6. Raman spectra of (a) orange's pericarp, (b) malathion formulation (40\% w/w) left on the surface of orange and (c) standard malathion solution

\section{Conclusions}

The micro-Raman spectra of apples, pears, oranges, several pesticides and pesticide residues on the surface of oranges were successfully recorded using a micro-Raman spectrometer. The characteristic peaks of pesticides can be seen from the spectra of pesticides left on the surface of fruits. Micro-Raman spectroscopy is a potential tool to determine the pesticide residues for fruit quality and safety control with rapid, non-destructive, low cost and environmentally friendly advantages. 


\section{Acknowledgements}

The authors gratefully acknowledge the financial support provided by National Science and Technology Support Program (2008BAD96B04), Natural Science Foundation of Jiangxi Province (2008GQN0029, 2007GZN0266), Special Science and Technology-Support Program for Foreign Science and Technology Cooperation Plan (2009BHB15200), Technological expertise and academic leaders training program of Jiangxi Province (2009DD00700).

\section{References}

1. UN Food and Agricultural Organization: International Code of Conduct on the Distribution and Use of Pesticides, Rome, Italy (2002)

2. Tuormaa, T.E.: Adverse Effects of Agrochemicals on Reproduction and Health: a Brief Review from the Literature. J. Nutritional Environ. Med. 5, 353-366 (1995)

3. Picó, Y., Font, G., Moltó, J.C., Mañes, J.: Pesticide Residue Determination in Fruit and Vegetables by Liquid Chromatography-mass Spectrometry. J. Chromatogr. A 882, 153-173 (2000)

4. Jansson, C., Pihlström, T., Österdahl, B., Markides, K.E.: A New Multi-residue Method for Analysis of Pesticide Residues in Fruit and Vegetables Using Liquid Chromatography with Tandem Mass Spectrometric Detection. J. Chromatogr. A 1023, 93-104 (2004)

5. Frenich, A.G., Vidal, J.L.M., López, T.L., Aguado, S.C., Salvador, I.M.: Monitoring Multi-class Pesticide Residues in Fresh Fruits and Vegetables by Liquid Chromatography with Tandem Mass Spectrometry. J. Chromatogr. A 1048, 199-206 (2004)

6. Guan, H.X., Brewer, W.E., Garris, S.T., Morgan, S.L.: Disposable Pipette Extraction for the Analysis of Pesticides in Fruit and Vegetables Using Gas Chromatography/Mass Spectrometry. J. Chromatogr. A 1217, 1867-1874 (2010)

7. Juan-García, A., Font, G., Juan, C., Picó, Y.: Pressurised Liquid Extraction and Capillary Electrophoresis-mass Spectrometry for the Analysis of Pesticide Residues in Fruits from Valencian Markets, Spain. Food Chem. 120, 1242-1249 (2010)

8. Lacina, O., Urbanova, J., Poustka, J., Hajslova, J.: Identification/Quantification of Multiple Pesticide Residues in Food Plants by Ultra-high-performance Liquid Chromatography-time-of-flight Mass Spectrometry. J. Chromatogr. A 1217, 648-659 (2010)

9. Armenta, S., Quintás, G., Garrigues, S., de la Guardia, M.: Mid-infrared and Raman Spectrometry for Quality Control of Pesticide Formulations. Trends Anal. Chem. 24, 772-781 (2005)

10. Shende, C.S., Inscore, F., Gift, A., Maksymiuk, P., Farquharson, S.: Analysis of Pesticides on or in Fruit by Surface-enhanced Raman Spectroscopy. In: Proc. SPIE, vol. 5587, pp. 170-176 (2004)

11. Zhou, X.F., Fang, Y., Zhang, P.X.: Raman Spectra of Pesticides on the Surface of Fruits. J. Light Scatt. 16, 11-14 (2004)

12. Zhang, P.X., Zhou, X.F., Cheng, A.Y.S., Fang, Y.: Raman Spectra from Pesticides on the Surface of Fruits. J. Phys. 28, 7-11 (2006)

13. Sudheer, S.K., Pillai, V.P.M., Nayar, V.U.: Characterization of Laser Processing of Single-crystal Natural Diamonds Using Micro-Raman Spectroscopic Investigations. J. of Raman Spectrosc. 38, 427-435 (2007) 
14. Palanza, V., Martino, D.D., Paleari, A., Spinolo, G., Prosperi, L.: Micro-Raman Spectroscopy Applied to the Study of Inclusions within Sapphire. J. Raman Spectrosc. 39, 1007-1011 (2008)

15. Doherty, B., Miliani, C., Berghe, I.V., Sgamellotti, A., Brunetti, B.G.: Micro-Raman Spectroscopic Study of Artificially Aged Natural and Dyed Wool. J. Raman Spectrosc. 39, 638-645 (2008)

16. Colomban, P., Dinh, H.M., Riand, J., Prinsloo, L.C., Mauchamp, B.: Nanomechanics of Single Silkworm and Spider Fibres: a Raman and Micro-mechanical in Situ Study of the Conformation Change with Stress. J. Raman Spectrosc. 39, 1749-1764 (2008)

17. Rull, F., Martinez-Frias, J., Sansano, A., Medina, J., Edwards, H.G.M.: Comparative Micro-Raman Study of the Nakhla and Vaca Muerta Meteorites. J. Raman Spectrosc. 35, 497-503 (2004)

18. Łodziński, M., Wrzalik, R., Sitarz, M.: Micro-Raman Spectroscopy Studies of Some Accessory Minerals from Pegmatites of the Sowie Mts and Strzegom-Sobótka Massif, Lower Silesia, Poland. J. Mol. Struct., 744-747, 1017-1026 (2005)

19. El-Abassy, R.M., Donfack, P., Materny, A.: Rapid Determination of Free Fatty Acid in Extra Virgin Olive Oil by Raman Spectroscopy and Multivariate Analysis. J. Am. Oil Chem. Soc. 86, 507-511 (2009)

20. Skoulika, S.G., Georgiou, C.A., Polissiou, M.G.: FT-Raman Spectroscopy-Analytical Tool for Routine Analysis of Diazinon Pesticide Formulations. Talanta 51, 599-604 (2000)

21. Quintás, G., Garrigues, S., de la Guardia, M.: FT-Raman Spectrometry Determination of Malathion in Pesticide Formulations. Talanta 63, 345-350 (2004) 